\title{
Age-based model for metacarpophalangeal joint proprioception in elderly
}

\author{
This article was published in the following Dove Press journal: \\ Clinical Interventions in Aging \\ 6 April 2017 \\ Number of times this article has been viewed
}

\author{
Mike D Rinderknecht' \\ Olivier Lambercy' \\ Vanessa Raible ${ }^{2}$ \\ Joachim Liepert ${ }^{2}$ \\ Roger Gassert ${ }^{\prime}$
}

'Rehabilitation Engineering Laboratory, Department of Health Sciences and Technology, Institute of Robotics and Intelligent Systems, ETH Zurich, Zurich, Switzerland; ${ }^{2}$ Department of Neurorehabilitation, Kliniken Schmieder, Allensbach, Germany
Correspondence: Mike D Rinderknecht Rehabilitation Engineering Laboratory, Department of Health Sciences and Technology, Institute of Robotics and Intelligent Systems, ETH Zurich, Lengghalde 5, CH-8092 Zurich, Switzerland

$\mathrm{Tel}+4 \mid 445107238$

Email mike.rinderknecht@hest.ethz.ch

\begin{abstract}
Neurological injuries such as stroke can lead to proprioceptive impairment. For an informed diagnosis, prognosis, and treatment planning, it is essential to be able to distinguish between healthy performance and deficits following the neurological injury. Since there is some evidence that proprioception declines with age and stroke occurs predominantly in the elderly population, it is important to create a healthy reference model in this specific age group. However, most studies investigate age effects by comparing young and elderly subjects and do not provide a model within a target age range. Moreover, despite the functional relevance of the hand in activities of daily living, age-based models of distal proprioception are scarce. Here, we present a proprioception model based on the assessment of the metacarpophalangeal joint angle difference threshold in 30 healthy elderly subjects, aged 55-80 years (median: 63, interquartile range: 58-66), using a robotic tool to apply passive flexion-extension movements to the index finger. A two-alternative forced-choice paradigm combined with an adaptive algorithm to define stimulus magnitude was used. The mixed-effects model analysis revealed that aging has a significant, increasing effect on the difference threshold at the metacarpophalangeal joint, whereas other predictors (eg, tested hand or sex) did not show a significant effect. The adaptive algorithm allowed reaching an average assessment duration $<15$ minutes, making its clinical applicability realistic. This study provides further evidence for an age-related decline in proprioception at the level of the hand. The established age-based model of proprioception in elderly may serve as a reference model for the proprioceptive performance of stroke patients, or of any other patient group with central or peripheral proprioceptive impairments. Furthermore, it demonstrates the potential of such automated robotic tools as a rapid and quantitative assessment to be used in research and clinical settings.
\end{abstract}

Keywords: aging, difference threshold, hand function, joint position sense, MCP, robotic assessment, presbypropria, somatosensation

\section{Introduction}

Proprioception consists of limb position sense (sense of stationary position) and kinesthesia (sense of limb movement). ${ }^{1}$ Proprioceptive information originates from muscle spindle afferents, mechanoreceptors in joint capsules and cutaneous tactile receptors. ${ }^{2,3}$ The perception and correct interpretation of proprioceptive inputs is an essential prerequisite for many activities of daily living (ADL), ${ }^{4}$ such as haptic exploration of objects ${ }^{5}$ and grasping. ${ }^{6}$ Proprioceptive function can be impaired, for example, as a result of peripheral or central neurological injuries, as in about half of stroke patients. ${ }^{7,8}$ There is some evidence that proprioceptive impairments reduce the probability of functional recovery, ${ }^{9}$ which motivates an increased attention to assessing and treating proprioceptive deficits. To be able to quantify these impairments, normative data from the healthy population are required. There is some literature showing that 
proprioception in healthy subjects declines with increased age (also referred to as presbypropria, ${ }^{10}$ for review, Goble et al ${ }^{11}$ ). Although the effect of age-related proprioceptive changes in the lower limbs on postural control in the elderly has been studied widely, relatively few studies have focused on the upper limbs, despite their importance for ADL. ${ }^{4-6}$ The decline in proprioceptive performance in more proximal joints (ie, shoulder, elbow, and wrist) could be demonstrated in a set of different experiments ${ }^{12-18}$ using mostly matching paradigms. For more distal joints (ie, in the hand) there exist some inconsistent results. ${ }^{19-22}$ The age-related effect on proprioception is usually investigated by comparing a young group of healthy subjects with an elderly group of healthy subjects. However, the incidence of neurological injuries may vary depending on the age, as it is the case for stroke, where incidence by age doubles each decade after the age of $55 . .^{23}$ Thus, stroke predominantly affects the elderly population. Therefore, to differentiate, for example, between healthy aging and proprioceptive deficits following a stroke, it would be of higher clinical utility to have a detailed model of how proprioception is affected by age within the target age group. To create a valid and accurate reference model, it is essential to base it on outcome measures from reliable and quantitative assessments.

Proprioception is commonly assessed with clinical tests, such as the up-down test in which the finger or toe is moved passively and the patient has to report the direction of movement, ${ }^{1,24}$ or a recent extension to the latter, named dual joint position test, where two digits are simultaneously moved in the same or reverse direction, which was shown to be superior. ${ }^{25}$ However, these tests are administered manually and suffer from poor inter-rater reliability and sensitivity. ${ }^{26}$ Due to the use of ordinal scales, these tests do not provide a precise quantification of proprioceptive function, and the provided outcome measures cannot serve as a basis for a reference model.

With the development of novel methods combined with simple tools, it has become possible to create fine-graded scales. ${ }^{21,27,28}$ Since with robotic technology it is possible to reduce or prevent manual intervention of the experimenter (eg, repositioning of the limb) and to take advantage of the control and sensing capabilities of robotic devices, stimuli can be presented in a more reproducible and well-controlled manner. This is suggested to increase reliability as well as sensitivity $^{29}$ or may reduce flooring and ceiling effects by using continuous outcome measures. As a result, many different studies quantifying proprioception with the help of robotic tools in combination with various assessment paradigms have emerged..$^{12,22,30-38}$ These kinds of assessments would allow creating reference models of healthy performance as a function of age. However, so far there exists only a very limited number of models, namely, for arm position matching ${ }^{12}$ and grasp aperture discrimination using spherical objects. ${ }^{21}$ Hence, since different assessment paradigms may target particular aspects of proprioception, it is necessary to create models, specific to the proprioceptive task, describing healthy performance of a target age group.

The purpose of this study was to create a model of proprioceptive function at the metacarpophalangeal (MCP) joint of the index finger in healthy elderly subjects to be used as a reference model for the prospective evaluation of proprioceptive deficits following peripheral and central neurological injuries, such as stroke. The index finger is essential for most grasp types used in ADL, ${ }^{39-41}$ and flexion of the $\mathrm{MCP}$ is a strong contributor to a major synergy for natural grasp patterns. ${ }^{42}$ The MCP joint angle difference threshold or limen (DL) was assessed with an automated robotic tool using an adaptive procedure named Parameter Estimation by Sequential Testing (PEST) ${ }^{43}$ in a two-alternative forced-choice $(2 \mathrm{AFC})^{44}$ paradigm. The influence of age, sex, dominance of the tested hand, finger length, measurement order, and number of trials on the DL was examined. We hypothesized that the model would reveal a major influence of age on the DL, thus, demonstrating the sensitivity of the proposed assessment method and its suitability for research on proprioception as well as clinical settings for a more informed diagnosis, prognosis, and treatment planning after stroke or other neurological injuries.

\section{Methods \\ Subjects}

Thirty healthy elderly subjects ( $\mathrm{S} 01-\mathrm{S} 30,62.8 \pm 6.4$ years, range 55-80 years, 14 males and 16 females, 29 right and 1 left handed) completed the study. Average finger length (measured from the MCP joint to the tip of the index finger) was $97.3 \pm 7.2 \mathrm{~mm}$ (across both hands). Handedness was assessed with the Edinburgh Handedness Inventory. ${ }^{45}$ Subjects were excluded from the study if they had somatosensory or motor deficits affecting hand function, or any history of neurological (central or peripheral) or hand injury. Before participating in the experiment, all subjects provided written informed consent. Ethical approval was obtained from the institutional ethics committee of ETH Zurich and the University of Konstanz.

\section{Robotic apparatus}

The assessment of MCP joint proprioception was performed with the Robotic Sensory Trainer (Figure 1) previously used 
in a pilot study. ${ }^{36}$ This device can provide well-controlled and reproducible passive finger movements (flexion and extension) around the MCP joint through an actuated remote center of motion (RCM) mechanism. ${ }^{32}$ The index finger is attached by means of two Velcro ${ }^{\circledR}$ straps to a sliding finger carriage mounted on the RCM mechanism. The hand and forearm supports can be adjusted with 6 degrees of freedom to allow for a comfortable posture. The tested hand of the subject is occluded from vision by a touchscreen to avoid visual cues on finger position. The subject can provide feedback on perceived stimuli directly on the touch-screen placed above the tested hand by using the nontested hand. Based on LabVIEW (National Instruments, Austin, TX, USA), the assessment runs autonomously and does not require intervention of the experimenter once initiated.

\section{Experimental protocol}

Subjects were seated in front of the assessment apparatus, and forearm, hand and index finger were strapped to the device after adjusting the supports. The MCP joint was carefully aligned with the RCM indicated by the extension of the black arrow mounted on the device (Figure 1). The MCP joint position DL was assessed for the index fingers of both hands in randomized order within one experimental session.

Every trial consisted of two successive passive finger movements to different flexion angles of the MCP joint (two-interval design, Figure 1). Passive movements were induced by the robotic apparatus, always starting from the same resting position (with all finger segments aligned, indicated by the dashed line in Figure 1). Flexion movements and movements back to resting position lasted $1 \mathrm{~s}$ each, whereas the MCP flexion angle was maintained for $1.5 \mathrm{~s}$. According to the $2 \mathrm{AFC}$ paradigm, subjects were asked after each trial to indicate on the touchscreen, using the nontested hand, which of the two presented angular displacements was larger. No feedback on correctness of the answer was provided. The difference between the two angles was defined as positive and centered around the flexion reference of $20^{\circ}$. The difference was adjusted from trial to trial using the adaptive PEST algorithm ${ }^{43}$ in order to converge toward the DL. PEST is based on a set of heuristic rules taking the subject's responses to past stimuli into account, leading to smaller stimulus differences in case of high proportion of correct responses, and larger difference in case of low proportion of correct responses. The range of angular differences was limited to $\left(0^{\circ}, 40^{\circ}\right)$ due to the mechanical limitations of the device. The same starting parameters (first presented angular difference of $5.5^{\circ}$, and first decreasing or increasing step of $2^{\circ}$ ), termination conditions (minimum step of $\pm 0.1^{\circ}$, or 20 consecutive trials at the same level), and logarithmic mapping as reported for the previous pilot study $^{36}$ were used. A maximum of 120 trials was permitted in case of nonconvergence, in order to keep the assessment duration short. These parameter values were selected based on our prior experimental knowledge and experience.

\section{Data analysis}

In order to estimate the DL from the experimental data, the proportion of correct responses at stimulus levels x (ie, at differences between the consecutively presented angles) was fitted with the psychometric function $\psi(\mathrm{x})$ in (1) using a Maximum Likelihood criterion: ${ }^{46}$

$$
\psi(\mathrm{x} ; \alpha, \beta, \gamma, \lambda)=\gamma+(1-\gamma-\lambda) \mathrm{F}(\mathrm{x} ; \alpha, \beta)
$$

$\mathrm{F}(\mathrm{x})$ corresponds to a cumulative normal function with the inflection point at $\alpha$ and the slope $\beta$ at this point. The guessing rate $\gamma$ was set to 0.5 , according to the $2 \mathrm{AFC}$ paradigm, and the lapse rate $\lambda$ (taking into account stimulus-independent errors, or "lapses") was allowed to vary between 0 and 0.1 , to reduce estimation bias ${ }^{47}$ The DL is defined at $\mathrm{x}=\psi^{-1}(0.75)$. The Weber fraction K (DL divided by the reference angle, here $20^{\circ}$ ) is reported together with the group average DL in degrees. Such a hybrid procedure combining adaptive sampling procedures and fitting of parametrized functions, as described by Hall, ${ }^{48}$ allows estimating the DL even when the adaptive sequence (here PEST sequence) does not converge and terminate within a maximum number of trials. ${ }^{36}$
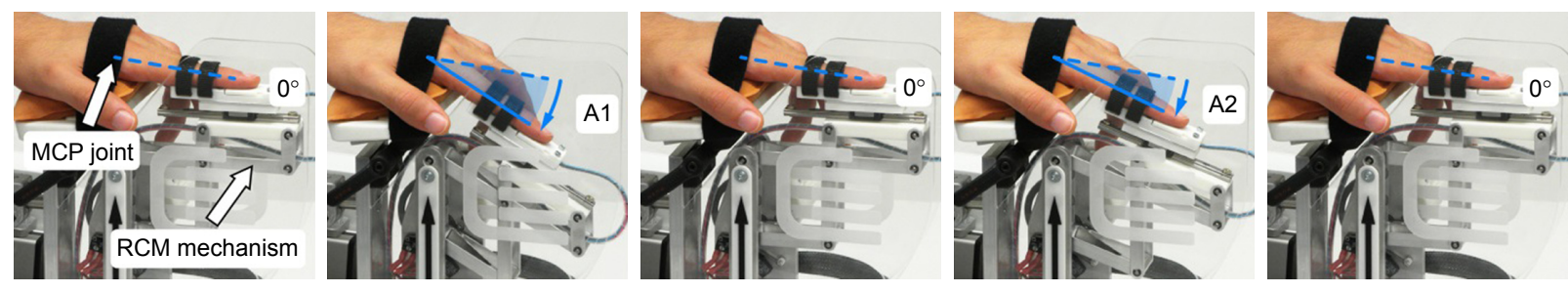

Figure I Side view on the RCM mechanism of the robotic device used to induce passive movements around the MCP joint.

Note: The sequence of pictures shows one trial, during which two different flexion angles (AI and A2) are presented.

Abbreviations: MCP, metacarpophalangeal; RCM, remote center of motion. 
A linear mixed-effects model analysis to describe the DL was performed. As fixed effects, age (in years), sex (male versus female), tested hand (dominant versus nondominant), index finger length (in millimeters), measurement order (first versus second assessment within the session), and number of trials were entered into the model. Furthermore, the interaction between the factors tested hand and measurement order was included. As random effects, intercepts for the subjects were added. Handedness (right versus left) was not included into the model, as the right and left handed groups were not balanced (29/1). A $\log _{10}$ transform was applied to the DL before fitting the model, since the DL is only supported in the positive, semi-infinite interval $(0, \infty)$. Visual inspection of residual plots did not reveal any obvious deviations from homoscedasticity or normality when using the $\log _{10}$ transform. Furthermore, the predictors were tested for collinearity. In order to assess the significance of fixed effects, the $P$-values were obtained by simulated $(\mathrm{n}=1,000)$ likelihood ratio tests (LRTs, MATLAB function compare) of the full model $\mathrm{H}_{\mathrm{A}}$ with the effect in question against the model $\mathrm{H}_{0}$ without the effect in question. This method generates a reference distribution of the LRT statistic $\mathrm{X}^{2}$ under $\mathrm{H}_{0}$ and compares it to the observed $\mathrm{X}^{2}$. This is computationally more intensive, but could be more accurate than comparing the test statistic $\mathrm{X}^{2}$ to a $\chi^{2}$-distribution using an analysis of variance, which is not always a very good approximation and tends to be anticonservative. To test whether clinical utility could be improved by reducing the maximum number of trials (120 to 60) to shorten the assessment duration, the same mixed-effects model analysis was conducted with truncated PEST sequences.

Significance levels were set to $\alpha=0.05$. Descriptive statistics are reported as mean \pm standard deviation (SD). All statistical analyses were performed in MATLAB R2014a (MathWorks, Natick, MA, USA).

\section{Results}

At group level (both hands), the MCP joint angle position DL averaged at $1.81^{\circ} \pm 0.96^{\circ}(\mathrm{K}=9.0 \% \pm 4.8 \%)$. On average, $65.3 \pm 27.3$ trials (ranging from 24 to 120 ) were required, resulting in an average duration of $14.3 \pm 6.0 \mathrm{~min}$ per assessment. In 5 out of the 60 assessments, the PEST algorithm did not converge, leading to a convergence rate of $91.7 \%$. The psychometric functions fitted to the proportion of correct responses and the evolution of the corresponding PEST sequences for both hands are shown for a representative subject (S22) in Figure 2. As visible in the top plots, stimulus levels are primarily sampled around the steeper part of the psychometric function, and the DL of the left, nondominant hand is smaller. In addition, only the assessment of the left, nondominant hand converged prior to 120 trials (bottom plots).

The mixed-effects model analysis revealed that age significantly affected the DL in increasing manner according to the simulated LRT $\left(P_{\text {age }}=0.029\right)$. The effect of age on the DL is illustrated in Figure 3 using averaged parameters of this sample multiplied by the estimates of the corresponding predictors not in question. Adding other fixed effects, that is, sex, finger length, handedness, tested hand, measurement, and number of trials as well as the interaction between tested hand and measurement, did not significantly improve the model for estimating the DL. Despite handedness not significantly improving the model, in 19 of 30 subjects, the DL of the nondominant hand was lower compared to the DL of the dominant hand. The simplest model for estimating the DL in healthy elderly (age range: 55-80 years) containing only the fixed effect age and a constant accounting for the other parameters was DL $=10^{(0.014 \text { age }-0.703)}$ in degrees. The complete summary of the mixed-effects model is provided in Table 1. When truncating the assessment to a maximum of 60 trials, the number of trials was $51.5 \pm 11.9$ on an average, with 33 of 60 assessments requiring all 60 trials. The changes in the model were minor $\left(\mathrm{DL}=10^{(0.015 \text { age }-0.704)}\right.$, simulated LRT $P_{\text {age }}=0.032$, all other $P$-values $\left.>0.05\right)$.

\section{Discussion}

This study aimed to create a model of MCP joint proprioception in the healthy elderly population to serve as a reference for patients suffering from proprioceptive deficits. Proprioception was quantified by the joint angle DL and assessed with a robotic tool applying well-controlled flexion movements to the index finger. As hypothesized, the proposed assessment approach is sensitive enough to capture a declining effect of $\mathrm{MCP}$ joint proprioception with increasing age. According to the model, the index finger MCP joint angle DL increases by around $2^{\circ}$ from age 55 to 80 . Furthermore, age was the only fixed effect having a significant influence on the DL, and it is thus essential to include it in a model of proprioception.

\section{Age-related decline of proprioception}

The observed age effect is consistent with most of the literature on proprioception at more proximal joints ${ }^{12-18}$ and distal joints. ${ }^{20-22}$ This decline in proprioception could be a consequence of increased proprioceptive attentional demand in older adults ${ }^{10}$ due to age-related central ${ }^{49}$ or peripheral ${ }^{12}$ physiological changes (for review, Goble et $\mathrm{al}^{11}$ ). However, there are also studies showing no age-related decline, ${ }^{16,19}$ or some showing age-related deficits depending on the task. ${ }^{50}$ 


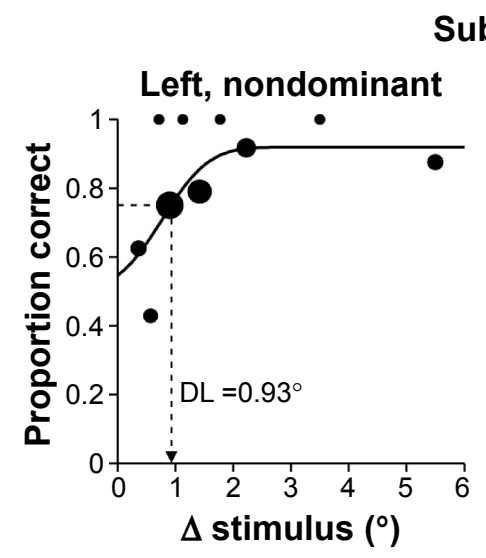

\section{Subject S22}
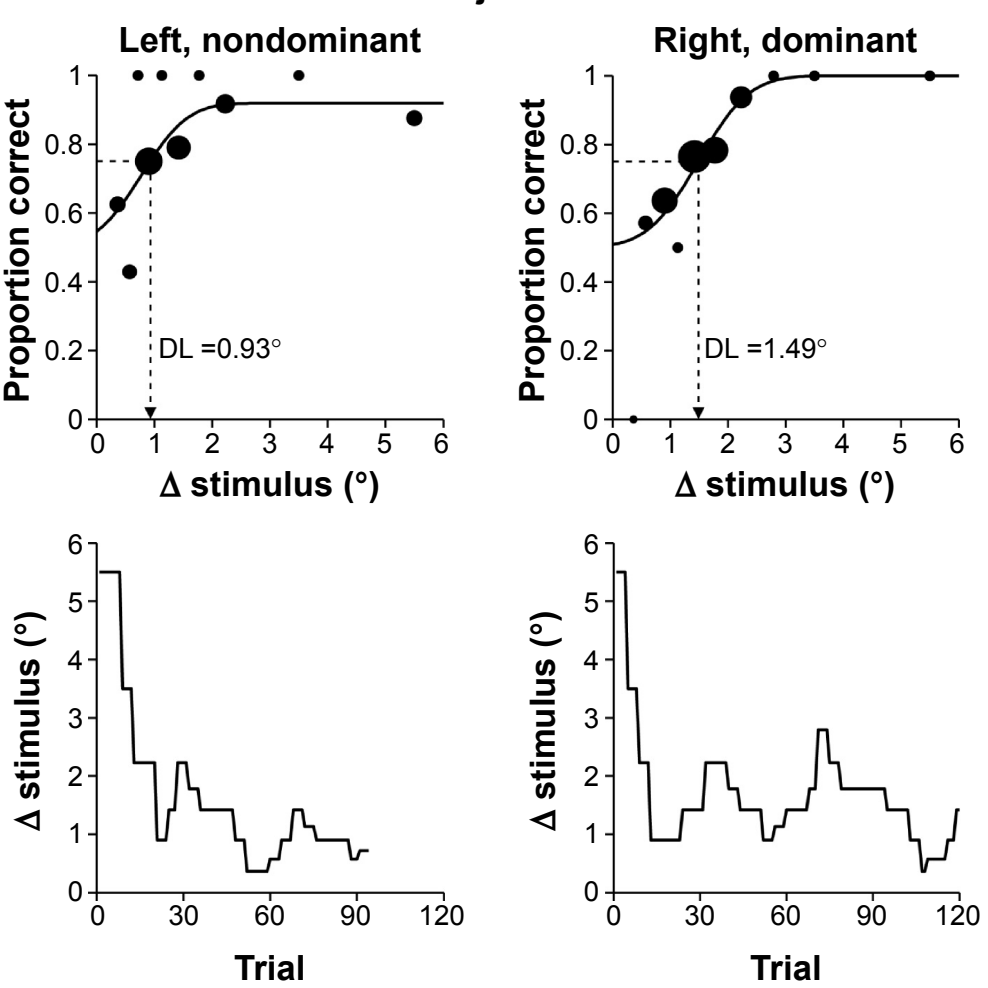

Figure 2 Resulting psychometric functions and PEST sequences of a representative subject.

Notes: (Top) Psychometric functions (thick lines) for both hands of subject S22 (67-year-old male). The size of the black dots indicates the number of presentations of a stimulus at a certain stimulus level ( $\Delta$ stimulus). (Bottom) Corresponding PEST sequences for the same subject. The thick line represents the stimulus level at each trial.

Abbreviations: DL, difference threshold or limen; PEST, Parameter Estimation by Sequential Testing.

At the level of the hand, the assessment of proximal interphalangeal joint position sense through position matching using a robotic tool that induced velocities below the movement detection threshold, reported a significant deterioration from the young to the elderly group, as well as a moderate, but significant, positive correlation $(r=0.466)$ between age and the magnitude of the matching error. ${ }^{20}$ Similarly, comparisons between different age groups in two proprioceptive tasks (indicating overlap of fingers during passive crisscross movements and onset of passive finger movement) revealed

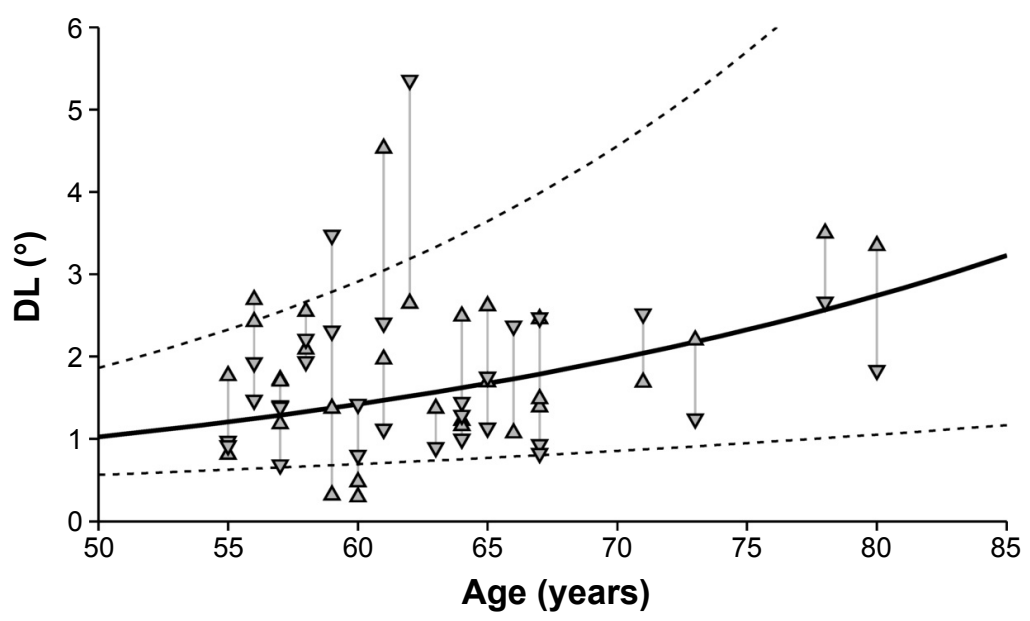

Figure 3 Age-DL relationship.

Notes: Aging has a significant increasing effect on the $D L(P<0.05)$. The $D L$ of the dominant hand is indicated by an upward-pointing triangle and the $D L$ of the nondominant by a downward-pointing triangle. Both assessments are connected through a gray vertical line for each subject. Black lines show the age-based $\mathrm{DL}$ model (thick line) $\pm \mathrm{SE}$ (dashed lines) obtained from the mixed-effects model analysis.

Abbreviations: DL, difference threshold or limen; SE, standard error. 
Table I Summary of the mixed-effects model analysis with the estimates and their SE predicting the $\log _{10} \mathrm{DL}$

\begin{tabular}{|c|c|c|c|c|c|c|c|c|}
\hline \multirow[t]{2}{*}{ Name } & \multirow[t]{2}{*}{ Estimate } & \multirow[t]{2}{*}{ SE } & \multirow[t]{2}{*}{$t$-Value } & \multirow[t]{2}{*}{ DF } & \multirow[t]{2}{*}{$P$-value } & \multicolumn{2}{|c|}{$\begin{array}{l}\text { Confidence } \\
\text { interval (95\%) }\end{array}$} & \multirow{2}{*}{$\begin{array}{l}\text { Simulated } \\
\text { LRT } \\
P \text {-value }\end{array}$} \\
\hline & & & & & & Lower & Upper & \\
\hline \multicolumn{9}{|l|}{ Fixed effects } \\
\hline (Intercept) & -0.857 & 0.678 & -1.27 & 52 & 0.212 & -2.217 & 0.503 & \\
\hline $\mathrm{Age}^{\mathrm{a}}$ & 0.014 & 0.005 & 2.74 & 52 & $0.008^{*}$ & 0.004 & 0.025 & $0.019 *$ \\
\hline Sex & 0.142 & 0.082 & 1.72 & 52 & 0.091 & -0.023 & 0.307 & 0.115 \\
\hline Finger length ${ }^{c}$ & -0.000 & 0.005 & -0.09 & 52 & 0.928 & -0.011 & 0.010 & 0.931 \\
\hline Trials $^{d}$ & 0.002 & 0.001 & 1.96 & 52 & 0.055 & -0.000 & 0.004 & 0.085 \\
\hline Tested hande & -0.054 & 0.085 & -0.64 & 52 & 0.527 & -0.224 & 0.116 & 0.801 \\
\hline Measurement ${ }^{f}$ & -0.010 & 0.083 & -0.12 & 52 & 0.903 & -0.177 & 0.157 & 0.724 \\
\hline Measurement*tested hand $d^{\text {e,f }}$ & 0.083 & 0.124 & 0.67 & 52 & 0.507 & -0.166 & 0.331 & 0.541 \\
\hline \multicolumn{9}{|l|}{ Random effects } \\
\hline Group & Name & SD & & & & & & \\
\hline Subject & (Intercept) & 0.048 & & & & & & \\
\hline Residual & & 0.213 & & & & & & \\
\hline
\end{tabular}

Notes: The last column shows the $P$-values from the simulated LRT. The fixed effect age affected the $D L$ significantly in increasing direction $(* P<0.05)$. ${ }^{a} B a s e l i n e ~=0$ years. ${ }^{\mathrm{b} B a s e l i n e}=$ male. ${ }^{\mathrm{C} B a s e l i n e}=0 \mathrm{~mm}$. ${ }^{\mathrm{d}}$ Baseline $=0$ trials. ${ }^{\mathrm{e} B a s e l i n e}=$ dominant. ${ }^{\mathrm{C} B a s e l i n e}=$ first.

Abbreviations: DF, degrees of freedom; DL, difference threshold or limen; LRT, likelihood ratio test; SD, standard deviation; SE, standard error.

larger errors in the elderly. In addition, an increase of 0.4 decision errors/decade for a total of 21 decisions was reported for an assessment where subjects had to indicate whether they perceived a comparison polystyrene ball to be larger, smaller, or equal to the volume of reference polystyrene ball. ${ }^{21}$ In contrast, a study also assessing proprioception at the MCP joint, but by determining the detection threshold of sinusoidal movements, reported no major age-related decline in joint motion sensation. ${ }^{19}$ However, it is difficult to compare the different findings on age-related changes in proprioception quantitatively due to the diversity of outcome measures arising from the different experimental paradigms. Models reported in the literature are based on matching paradigms and provide matching errors, ${ }^{12,20,21}$ which cannot be directly related to different thresholds of proprioceptive perception.

One limitation of this study is the fact that the created model is limited to an age range of 55-80 years, and that the recruited subjects were not uniformly distributed across age. In particular, the number of subjects with age $>70$ years was small. However, additional verification of the model parameters showed that the model was robust to exclusion of the four most elderly subjects ( $>70$ years), demonstrating that the effect of declining proprioception is supported by the entire data set. Furthermore, no comparison to a young group of healthy subjects was made. Yet, as expected, the group average of the DL in the elderly sample population of this study is slightly higher compared to the one reported for healthy young subjects $\left(1.73^{\circ}, \mathrm{K}=8.6 \%\right)$ in the pilot study using the same apparatus and practically identical protocol. ${ }^{36}$
Furthermore, although the incidence by age doubles each decade after the age of $55,{ }^{23}$ the age range of our study covers the major part of our target population. Although the comparison of young and elderly subjects can support the investigation of age-related changes, it has limited clinical value compared to an age-based reference model.

\section{Influence of hand dominance and sex}

Although the mixed-effects model did not show a significant effect of the tested hand (dominant versus nondominant) on the DL, about two-thirds of the subjects showed a better performance (ie, lower DL) with the nondominant limb. This trend is well in line with some literature, suggesting proprioceptive processing advantages of the nondominant limb in some conditions, ${ }^{12,16,51-55}$ while others suggest gain differences of sensory-motor loops as an explanation. ${ }^{56}$ In contrast to those studies, other groups did not identify any difference between dominant and nondominant limb. ${ }^{18,22}$ There are also studies showing smaller matching errors with the dominant limb. ${ }^{28}$ It has been suggested that long-term use-dependent superiority of the dominant hand may enhance proprioception. ${ }^{57}$ As a conclusion, proprioception may be superior in the nondominant limb, although the dominant hand is generally more dexterous. However, as the results from the different studies show, this may strongly depend on the assessment paradigm used, as motor function may be a strong confound in some assessments, as for example in active matching tasks.

As in most previous studies, we did not find an effect of sex on proprioception. ${ }^{16,28,51,58}$ There was one study that 
identified sex-related differences, however, only in some of the outcome measures of a matching task including movements of the elbow and shoulder. ${ }^{12}$

\section{Robustness to fatigue and learning, number of trials, and clinical utility}

Based on the results from this study, the DL is robust with respect to the measurement order (first versus second of two consecutive assessments, also if hand dominance is taken into account) and the number of trials. Whether the dominant/nondominant hand was assessed first or second within the session had no influence on the DL. This suggests that there is no learning effect of the task, which is essential for an assessment, as the goal is to measure the capacity of the subject and not the improvement due to increased level of familiarization with the task. Despite both hands being assessed consecutively, no fatigue effect could be observed. It could be, though, that learning and fatigue effects cancel each other. However, the fact that a larger number of trials does not have a significant influence on the DL either, provide further evidence that there is no important fatigue effect.

As previously shown in a pilot study with healthy young subjects, the adaptive sampling procedure PEST allows reducing the assessment time considerably compared to the widely used, but inefficient, method of constant stimuli, ${ }^{36}$ resulting in an average assessment duration of $\sim 15 \mathrm{~min}$. Truncating PEST sequences to a maximum of 60 trials resulted in an average assessment duration of $11 \mathrm{~min}$ and only in minor changes of the mixed-effects model, demonstrating robustness of the assessment. This is crucial because shortening the assessment duration can significantly increase the assessment's clinical utility, especially for patient groups, where assessment time is expensive, and where time constraints are perceived as a barrier. ${ }^{59}$ Furthermore, reliability and validity of an assessment could benefit from short assessments, as the influence of confounding factors such as inattention and other cognitive factors would be decreased. ${ }^{60}$

\section{Advantages and limitations of the assessment paradigm}

This paradigm assesses proprioception in an isolated way, in contrast to assessments using ipsilateral and contralateral matching tasks, which most often require the subject to move actively to reproduce a presented position. Thus, this assessment can be used to investigate somatosensory deficits independently of motor deficits. This allows investigating their contribution to functional impairments and effect on recovery after neurological injuries, such as stroke.

Besides potential confounding factors such as learning and fatigue, the 2AFC method could also be affected by short-term memory. Previous studies have shown that absolute errors in position matching tasks are significantly influenced by several factors such as the type of position matching task or reference position establishment (eg, reference joint angle and how the limb was displaced to present this angle). ${ }^{61}$ A study with 10 healthy subjects demonstrated better proprioceptive performance in an ipsilateral matching task requiring short-term memory than in a contralateral (simultaneous) matching task requiring interhemispheric transfer, which in turn is better than in a contralateral remembered task requiring both, memory and interhemispheric transfer. ${ }^{61}$ Similar results have been presented in another study for these three task types revealing mostly disproportionate increases in matching errors for the contralateral remembered task. ${ }^{15}$ A parallel can be drawn to the assessments of difference thresholds using either two intervals (as was the case here) requiring short-term memory (the two stimuli have to be remembered and compared postpresentation) or two locations stimulated simultaneously (eg, on both index fingers) requiring interhemispheric transfer for comparison, inducing temporal or spatial errors, respectively. Hence, this suggests that the paradigm used in this study (two-interval 2AFC) should be less affected by factors besides healthy aging of proprioception and also be less error prone, as no interhemispheric transfer is required. Furthermore, many limb matching tests rely on the sensorimotor function of the ipsilesional "unimpaired" limb of the patient, which may also be affected by a cerebral lesion, ${ }^{8,62}$ and their outcomes might also be influenced by deficits in the central integration of proprioceptive information across the two limbs. These confounds are also fully addressed by the two-interval $2 \mathrm{AFC}$ approach. Moreover, compared to other psychophysical paradigms such as Yes-No, Remainder, and Same-Different, the $2 \mathrm{AFC}$ approach is more robust against decision criteria (ie, response bias), and thus more objective. ${ }^{44,63}$

Although in each trial of the $2 \mathrm{AFC}$ assessment paradigm movements with different amplitudes are presented, there is a trade-off between constant movement duration versus constant movement velocity. Thus, subjects could rely on one of these potential confounds besides the presented joint position angle. There have been approaches where a subthreshold movement velocity was used to resolve this trade-off. ${ }^{20}$ However, this approach is only usable in research, as it leads to an increased trial duration resulting in overly long 
assessments, thus limiting their clinical applicability. For our assessment, a constant duration with varying velocity was chosen because perception of movement velocity (ie, kinesthesia) is a subpart of proprioception, and both position and velocity information is suggested to be incorporated within internal models. ${ }^{64}$ In contrast, discriminating time intervals, apart from detection of movement onset and cessation following discharge of muscle spindles, ${ }^{65}$ is not strictly part of the proprioceptive sense.

\section{Conclusion}

The results of this study demonstrate that taking age into account when creating a model of healthy performance of proprioception as a reference for neurological patients suffering from proprioceptive deficits is essential. All the more, as in some neurological injuries, such as stroke, the ipsilesional "unimpaired" limb of the patient can also be potentially affected ${ }^{8,62}$ and should thus not be used as a reference to quantify the performance of the impaired limb. ${ }^{66}$ Furthermore, this study supports the clinical utility of the objective and automated assessment approach using a robotic tool for quantifying the angular DL at the MCP joint through its sensitivity and rapid administration. This highlights its potential as an assessment tool to be used in combination with age-based reference models for an informed diagnosis, prognosis, and planning of clinical interventions.

\section{Acknowledgments}

The authors thank J-C Metzger and WL Popp for their valuable suggestions and thoughtful discussions, as well as the statistical consulting service at ETH Zurich for their advice. This research was supported by the National Center of Competence in Research on Neural Plasticity and Repair of the Swiss National Science Foundation, the Janggen-Pöhn Foundation, ETH Zurich and the Stiftung Schmieder für Wissenschaft und Forschung.

\section{Disclosure}

The authors report no conflicts of interest in this work.

\section{References}

1. Gilman S. Joint position sense and vibration sense: anatomical organisation and assessment. J Neurol Neurosurg Psychiatry. 2002;73(5): 473-477.

2. Gandevia SC, McCloskey DI. Joint sense, muscle sense, and their combination as position sense, measured at the distal interphalangeal joint of the middle finger. J Physiol. 1976;260(2):387-407.

3. Gardner E, Martin J. The bodily senses. In: Kandel ER JT, editor. Principles of Neural Science. New York: McGraw-Hill; 2000: 430-450.

4. McPhee SD. Functional hand evaluations: a review. Am J Occup Ther. 1987;41(3):158-163.
5. Overvliet KE, Smeets JB, Brenner E. The use of proprioception and tactile information in haptic search. Acta Psychol (Amst). 2008; 129(1):83-90.

6. Gentilucci M, Toni I, Chieffi S, Pavesi G. The role of proprioception in the control of prehension movements: a kinematic study in a peripherally deafferented patient and in normal subjects. Exp Brain Res. 1994;99(3):483-500.

7. Connell LA, Lincoln NB, Radford KA. Somatosensory impairment after stroke: frequency of different deficits and their recovery. Clin Rehabil. 2008;22(8):758-767.

8. Carey LM, Matyas TA. Frequency of discriminative sensory loss in the hand after stroke in a rehabilitation setting. J Rehabil Med. 2011; 43(3):257-263.

9. Reding MJ, Potes E. Rehabilitation outcome following initial unilateral hemispheric stroke. Life table analysis approach. Stroke. 1988;19(11):1354-1358.

10. Boisgontier MP, Olivier I, Chenu O, Nougier V. Presbypropria: the effects of physiological ageing on proprioceptive control. Age (Dordr). 2012;34(5):1179-1194.

11. Goble DJ, Coxon JP, Wenderoth N, Van Impe A, Swinnen SP. Proprioceptive sensibility in the elderly: degeneration, functional consequences and plastic-adaptive processes. Neurosci Biobehav Rev. 2009;33(3): 271-278.

12. Herter TM, Scott SH, Dukelow SP. Systematic changes in position sense accompany normal aging across adulthood. J Neuroeng Rehabil. 2014;11:43.

13. Stelmach G, Sirica A. Aging and proprioception. AGE. 1986;9(4): 99-103.

14. Fry-Welch D, Campbell J, Foltz B, Macek R. Age-related changes in upper extremity kinesthesis. Phys Occup Ther Geriatr. 2003;20(3-4): 137-154.

15. Adamo DE, Martin BJ, Brown SH. Age-related differences in upper limb proprioceptive acuity. Percept Mot Skills. 2007;104(3 Pt 2): 1297-1309.

16. Schmidt L, Depper L, Kerkhoff G. Effects of age, sex and arm on the precision of arm position sense-left-arm superiority in healthy righthanders. Front Hum Neurosci. 2013;7:915.

17. Adamo DE, Alexander NB, Brown SH. The influence of age and physical activity on upper limb proprioceptive ability. J Aging Phys Act. 2009; 17(3):272-293.

18. Wright ML, Adamo DE, Brown SH. Age-related declines in the detection of passive wrist movement. Neurosci Lett. 2011;500(2):108-112.

19. Kokmen E, Bossemeyer R, Williams WJ. Quantitative evaluation of joint motion sensation in an aging population. J Gerontol. 1978; 33(1):62-67.

20. Ferrell WR, Crighton A, Sturrock RD. Age-dependent changes in position sense in human proximal interphalangeal joints. Neuroreport. 1992;3(3):259-261.

21. Kalisch T, Kattenstroth JC, Kowalewski R, Tegenthoff M, Dinse HR. Age-related changes in the joint position sense of the human hand. Clin Interv Aging. 2012;7:499-507.

22. Ingemanson ML, Rowe JB, Chan V, Wolbrecht ET, Cramer SC, Reinkensmeyer DJ. Use of a robotic device to measure age-related decline in finger proprioception. Exp Brain Res. 2015;234(1):83-93.

23. Feigin VL, Lawes CM, Bennett DA, Anderson CS. Stroke epidemiology: a review of population-based studies of incidence, prevalence, and casefatality in the late 20th century. Lancet Neurol. 2003;2(1):43-53.

24. Epstein O, Perkin GD, Cookson J, et al. Clinical Examination. London: Elsevier Health Sciences; 2008.

25. Beckmann YY, Çiftçi Y, Ertekin C. The detection of sensitivity of proprioception by a new clinical test: the dual joint position test. Clin Neurol Neurosurg. 2013;115(7):1023-1027.

26. Lincoln NB, Crow JL, Jackson JM, Waters GR, Adams SA, Hodgson P. The unreliability of sensory assessments. Clin Rehabil. 1991;5(4): 273-282.

27. Carey LM, Oke LE, Matyas TA. Impaired limb position sense after stroke: a quantitative test for clinical use. Arch Phys Med Rehabil. 1996; 77(12):1271-1278. 
28. Wycherley AS, Helliwell PS, Bird HA. A novel device for the measurement of proprioception in the hand. Rheumatology (Oxford). 2005;44(5):638-641.

29. Scott SH, Dukelow SP. Potential of robots as next-generation technology for clinical assessment of neurological disorders and upperlimb therapy. J Rehabil Res Dev. 2011;48(4):335-353.

30. Dukelow SP, Herter TM, Moore KD, et al. Quantitative assessment of limb position sense following stroke. Neurorehabil Neural Repair. 2010;24(2):178-187.

31. Squeri V, Zenzeri J, Morasso P, Basteris A. Integrating proprioceptive assessment with proprioceptive training of stroke patients. In: Rehabilitation Robotics (ICORR), 2011 IEEE International Conference on (Zurich, Switzerland):1-6, 2011.

32. Lambercy O, Juárez Robles A, Kim Y, Gassert R. Design of a robotic device for assessment and rehabilitation of hand sensory function. In: Rehabilitation Robotics (ICORR), 2011 IEEE International Conference on (Zurich, Switzerland):1-6, 2011.

33. Dukelow SP, Herter TM, Bagg SD, Scott SH. The independence of deficits in position sense and visually guided reaching following stroke. J Neuroeng Rehabil. 2012;9:72.

34. Semrau JA, Herter TM, Scott SH, Dukelow SP. Robotic identification of kinesthetic deficits after stroke. Stroke. 2013;44(12):3414-3421.

35. Simo L, Botzer L, Ghez C, Scheidt RA. A robotic test of proprioception within the hemiparetic arm post-stroke. J Neuroeng Rehabil. 2014; 11:77.

36. Rinderknecht MD, Popp WL, Lambercy O, Gassert R. Experimental Validation of a Rapid, Adaptive Robotic Assessment of the MCP Joint Angle Difference Threshold. In: Auvray M, Duriez C, editors. Haptics: Neuroscience, Devices, Modeling, and Applications Lecture Notes in Computer Science. Berlin: Heidelberg Springer; 2014:3-10.

37. Cappello L, Elangovan N, Contu S, Khosravani S, Kolchak J, Maisa L. Robot-aided assessment of wrist proprioception. Front Hum Neurosci. 2015;9:198.

38. Rinderknecht MD, Popp WL, Lambercy O, Gassert R. Reliable and rapid robotic assessment of wrist proprioception using a gauge position matching paradigm. Front Hum Neurosci. 2016;10:316.

39. Elliott JM, Connolly KJ. A classification of manipulative hand movements. Dev Med Child Neurol. 1984;26(3):283-296.

40. Felix T, Romero J, Schiedam HB, Dollar AM, Kragic D. The GRASP taxonomy of human grasp types. IEEE Trans Hum-Mach Syst. 2016; 46(1):66-77.

41. Kamakura N, Matsuo M, Ishii H, Mitsuboshi F, Miura Y. Patterns of static prehension in normal hands. Am J Occup Ther. 1980;34(7):437-445.

42. Santello M, Flanders M, Soechting JF. Postural hand synergies for tool use. J Neurosci. 1998;18(23):10105-10115.

43. Taylor MM, Douglas Creelman C. PEST: efficient estimates on probability functions. J Acoust Soc Am. 1967;41(4):782.

44. Macmillan NA, Douglas Creelman C. Detection theory: a User's Guide. New Jersey: Lawrence Erlbaum Associates; 2005.

45. Oldfield RC. The assessment and analysis of handedness: the Edinburgh inventory. Neuropsychologia. 1971;9(1):97-113.

46. Prins N, Kingdom FAA. Palamedes: Matlab routines for analyzing psychophysical data (version 1.8.2); 2009.

47. Wichmann FA, Hill NJ. The psychometric function: I. Fitting, sampling, and goodness of fit. Percept Psychophys. 2001;63(8):1293-1313.
48. Hall JL. Hybrid adaptive procedure for estimation of psychometric functions. J Acoust Soc Am. 1981;69(6):1763-1769.

49. Goble DJ, Coxon JP, Van Impe A, et al. The neural basis of central proprioceptive processing in older versus younger adults: an important sensory role for right putamen. Hum Brain Mapp. 2012;33(4):895-908.

50. Boisgontier MP, Swinnen SP. Age-related deficit in a bimanual joint position matching task is amplitude dependent. Front Aging Neurosci. 2015;7:162.

51. Goble DJ, Lewis CA, Brown SH. Upper limb asymmetries in the utilization of proprioceptive feedback. Exp Brain Res. 2006;168(1-2): 307-311.

52. Goble DJ, Brown SH. The biological and behavioral basis of upper limb asymmetries in sensorimotor performance. Neurosci Biobehav Rev. 2008;32(3):598-610.

53. Goble DJ, Brown SH. Upper limb asymmetries in the matching of proprioceptive versus visual targets. J Neurophysiol. 2008;99(6): 3063-3074.

54. Goble DJ, Brown SH. Dynamic proprioceptive target matching behavior in the upper limb: effects of speed, task difficulty and arm/hemisphere asymmetries. Behav Brain Res. 2009;200(1):7-14.

55. Goble DJ, Noble BC, Brown SH. Proprioceptive target matching asymmetries in left-handed individuals. Exp Brain Res. 2009;197(4): 403-408.

56. Adamo DE, Martin BJ. Position sense asymmetry. Exp Brain Res. 2009;192(1):87-95.

57. Teixeira LA. Categories of manual asymmetry and their variation with advancing age. Cortex. 2008;44(6):707-716.

58. Djupsjöbacka M, Domkin D. Correlation analysis of proprioceptive acuity in ipsilateral position-matching and velocity-discrimination. Somatosens Mot Res. 2005;22(1-2):85-93.

59. Pumpa LU, Cahill LS, Carey LM. Somatosensory assessment and treatment after stroke: an evidence-practice gap. Aust Occup Ther J. 2015; 62(2):93-104.

60. Goble DJ, Mousigian MA, Brown SH. Compromised encoding of proprioceptively determined joint angles in older adults: the role of working memory and attentional load. Exp Brain Res. 2012;216(1):35-40.

61. Goble DJ. Proprioceptive acuity assessment via joint position matching: from basic science to general practice. Phys Ther. 2010;90(8): 1176-1184.

62. Schaefer SY, Haaland KY, Sainburg RL. Ipsilesional motor deficits following stroke reflect hemispheric specializations for movement control. Brain. 2007;130(Pt 8):2146-2158.

63. Gescheider G. Psychophysics: Method, Theory, and Applications. New Jersey: Lawrence Erlbaum Associates; 1985.

64. Kerr GK, Worringham CJ. Velocity perception and proprioception. Adv Exp Med Biol. 2002;508:79-86.

65. Grill SE, Hallett M. Velocity sensitivity of human muscle spindle afferents and slowly adapting type II cutaneous mechanoreceptors J Physiol. 1995;489(Pt 2):593.

66. Dannenbaum RM, Jones LA. The assessment and treatment of patients who have sensory loss following cortical lesions. J Hand Ther. 1993 ; 6(2):130-138
Clinical Interventions in Aging

\section{Publish your work in this journal}

Clinical Interventions in Aging is an international, peer-reviewed journal focusing on evidence-based reports on the value or lack thereof of treatments intended to prevent or delay the onset of maladaptive correlates of aging in human beings. This journal is indexed on PubMed Central, MedLine,

\section{Dovepress}

CAS, Scopus and the Elsevier Bibliographic databases. The manuscript management system is completely online and includes a very quick and fair peer-review system, which is all easy to use. Visit http://www.dovepress. com/testimonials.php to read real quotes from published authors. 